\title{
Morse subsets of CAT(0) spaces are strongly contracting
}

\section{Christopher H. Cashen ${ }^{1}$}

Received: 7 October 2018 / Accepted: 7 May 2019 / Published online: 10 May 2019

(c) The Author(s) 2019

\begin{abstract}
We prove that Morse subsets of CAT(0) spaces are strongly contracting. This generalizes and simplifies a result of Sultan, who proved it for Morse quasi-geodesics. Our proof goes through the recurrence characterization of Morse subsets.
\end{abstract}

Keywords Morse set · Contracting set $\cdot$ Recurrent set $\cdot$ CAT(0) space $\cdot$ Strongly contracting Mathematics Subject Classification (2010) 20F65 · 20F67

In this note we give a short proof of the following technical result:

Proposition 1 If $\mathcal{Z}$ is a $\rho$-recurrent subset of a $C A T(0)$ space $\mathcal{X}$ and the empty set is not in the image of the map $\pi_{\mathcal{Z}}(x):=\{z \in \mathcal{Z} \mid d(x, z)=d(x, \mathcal{Z})\}$ then $\mathcal{Z}$ is $12 \rho(21)$-strongly contracting.

This is the final piece of the following theorem, which says that a number of properties that are equivalent to quasi-convexity in hyperbolic spaces are also equivalent to one another in $\mathrm{CAT}(0)$ spaces:

Theorem 1 Let $\mathcal{X}$ be a geodesic metric space. Let $\mathcal{Z}$ be an unbounded subset of $\mathcal{X}$ such that the empty set is not in the image of $\pi_{\mathcal{Z}}$. The following are equivalent:

$\mathcal{Z}$ is Morse $\quad$ There is a function $\mu:[1, \infty) \times[0, \infty) \rightarrow[0, \infty)$ defined by $\mu(L, A):=\sup _{\gamma} \sup _{w \in \gamma} d(w, \mathcal{Z})$, where the first supremum is taken over $(L, A)$-quasi-geodesic segments $\gamma$ with both endpoints on $\mathcal{Z}$.

$\mathcal{Z}$ is contracting There is a function $\sigma:[0, \infty) \rightarrow[0, \infty)$ with $\lim _{r \rightarrow \infty} \sigma(r) / r=0$ defined by:

$$
\sigma(r):=\sup _{d(x, y) \leq d(x, \mathcal{Z}) \leq r} \operatorname{diam} \pi_{\mathcal{Z}}(x) \cup \pi_{Z}(y)
$$

$\mathcal{Z}$ is recurrent $\quad$ There is a function $\rho:[1, \infty) \rightarrow[0, \infty)$ defined by:

$$
\rho(q):=\sup _{\Delta(\gamma) \leq q} \inf _{w \in \gamma} d\left(w, \mathcal{Z}^{\prime}\right)
$$

This research was supported by the Austrian Science Fund (FWF): P 30487-N35.

$凶$ Christopher H. Cashen

christopher.cashen@univie.ac.at

1 Faculty of Mathematics, University of Vienna, 1090 Vienna, Austria 
The first supremum is taken over rectifiable segments $\gamma$ with distinct endpoints on $\mathcal{Z}$ such that $\Delta(\gamma):=\frac{\text { len }(\gamma)}{d\left(\gamma^{+}, \gamma^{-}\right)} \leq q$, where $\gamma^{+}$and $\gamma^{-}$are the endpoints of $\gamma$ and $\mathcal{Z}^{\prime}$ is $\mathcal{Z}$ with the open balls of radius $d\left(\gamma^{+}, \gamma^{-}\right) / 3$ about $\gamma^{+}$and $\gamma^{-}$removed.

If $\mathcal{X}$ is hyperbolic or $C A T(0)$ then these conditions are equivalent to:

\section{$\mathcal{Z}$ is strongly contracting $\mathcal{Z}$ is contracting and the contraction gauge $\sigma$ is a bounded function.}

\section{Corollary 1 Morse subsets of CAT(O) spaces are strongly contracting.}

We refer the reader to [3] for background on hyperbolic and CAT(0) spaces.

The Proposition and the Theorem can be extended to arbitrary non-empty subsets $\mathcal{Z}$ by suitable modification of the definitions. Specifically, if the empty set is in the image of $\pi_{Z}$ then redefine $\pi_{\mathcal{Z}}(x):=\{z \in \mathcal{Z} \mid d(x, z) \leq d(x, \mathcal{Z})+1\}$. Extra bookkeeping is then required to compute an explicit contraction bound in the proof of the proposition. For bounded sets the four properties are trivially satisfied, with the possible exception that the given definition of recurrence does not make sense if some $\mathcal{Z}^{\prime}$ is empty, which occurs, for instance, when $\mathcal{Z}$ is a two point set. We could redefine $\rho$ to be the diameter of $\mathcal{Z}$ in this case.

The corollary confirms a conjecture of Russell et al. [7] and generalizes a result of Sultan [8], who proved that Morse quasi-geodesics in CAT( 0$)$ spaces are strongly contracting.

Genevois [6] proved that Morse subsets of a finite dimensional CAT(0) cube complex $\mathcal{X}$ are strongly contracting in the combinatorial metric. While this is quasi-isometric to the $\mathrm{CAT}(0)$ metric, the property of being a strongly contracting subset is not, in general, preserved by quasi-isometries [2], so Genevois's result and our theorem are independent. However, since the Morse property is preserved by quasi-isometries, and since Morse equals strongly contracting in both metrics, $\mathcal{X}$ has the same strongly contracting subsets regardless of whether it is endowed with the $\operatorname{CAT}(0)$ or the combinatorial metric.

Proof of the theorem The contraction condition was introduced in [1], where it was shown to be equivalent to the Morse condition. The recurrence condition was used to characterize Morse quasi-geodesics in [5], and this characterization can be extended to arbitrary subsets, as in [4, Theorem 2.2]. Strong contraction obviously implies contraction. It is easy to see that all of these properties are equivalent to quasi-convexity in hyperbolic spaces. The proposition supplies the remaining implication.

There is extensive literature making use of the Morse property and equivalent characterizations in various settings, but a complete exposition would be longer than this paper, so we will not attempt it. Sultan's result uses a characterization of the images of Morse quasi-geodesics in asymptotic cones due to Druţu et al. [5]. Loosely speaking, this characterization depends on there being a sensible notion of one point being between two others, which we have for quasi-geodesics but not, at least in an obvious way, for arbitrary subsets. We avoid the use of asymptotic cones and instead use recurrence (which also comes from [5]). We construct curves in essentially the same way as Sultan, but our argument, in addition to applying to general subsets, is simpler and gives an explicit strong contraction bound.

Proof of the proposition Define $D:=\rho(21)$. Supposing the contraction gauge $\sigma$ of $\mathcal{Z}$ is not bounded by $12 D$, we derive a contradiction. Failure of the contraction bound means there exist points $x, y \in \mathcal{X}$ such that $d(x, y) \leq d(x, \mathcal{Z})$ and such that diam $\pi_{\mathcal{Z}}(x) \cup \pi_{\mathcal{Z}}(y)>12 D$. We may assume $d(x, \mathcal{Z}) \geq d(y, \mathcal{Z})$, because otherwise $d(x, y) \leq d(y, \mathcal{Z})$ and we can swap 
the roles of $x$ and $y$. Choose $x^{\prime} \in \pi_{\mathcal{Z}}(x)$ and $y^{\prime} \in \pi_{\mathcal{Z}}(y)$ such that $P:=d\left(x^{\prime}, y^{\prime}\right)>12 D$. Let $\mathcal{Z}^{\prime}$ denote the set $\mathcal{Z}$ with the open balls of radius $P / 3$ about $x^{\prime}$ and $y^{\prime}$ removed.

For points $a, b \in \mathcal{X}$, let $[a, b]:[0,1] \rightarrow \mathcal{X}$ denote the geodesic segment from $a$ to $b$, parameterized proportional to arc length. Concatenation is denoted ' + '.

$$
\text { If } d\left(w, \mathcal{Z}^{\prime}\right) \leq D \text { for some } w \in \mathcal{X} \text { then } w \notin\left[x^{\prime}, x\right]+[x, y]+\left[y, y^{\prime}\right] .
$$

To see this, first suppose $w \in\left[x^{\prime}, x\right]$. Then $x^{\prime} \in \pi_{\mathcal{Z}}(w)$, so $P / 3 \leq d\left(x^{\prime}, \mathcal{Z}^{\prime}\right) \leq d\left(x^{\prime}, w\right)+$ $d\left(w, \mathcal{Z}^{\prime}\right)=d(w, \mathcal{Z})+d\left(w, \mathcal{Z}^{\prime}\right) \leq 2 d\left(w, \mathcal{Z}^{\prime}\right) \leq 2 D$, which is a contradiction, since $P>12 D$. Similarly, $w \notin\left[y^{\prime}, y\right]$. If $w \in[x, y]$ then:

$$
d(x, w)+d(w, y)=d(x, y) \leq d(x, \mathcal{Z}) \leq d(x, w)+D
$$

Thus, $d(w, y) \leq D$, which implies:

$$
P / 3 \leq d\left(y^{\prime}, \mathcal{Z}^{\prime}\right) \leq d\left(y^{\prime}, y\right)+d\left(y, \mathcal{Z}^{\prime}\right) \leq 2 d\left(y, \mathcal{Z}^{\prime}\right) \leq 2\left(d(y, w)+d\left(w, \mathcal{Z}^{\prime}\right)\right) \leq 4 D
$$

Again, this contradicts the hypothesis that $P>12 D$, so $\left(^{*}\right)$ is verified.

Now there are three cases to consider.

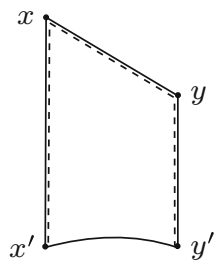

Case 1

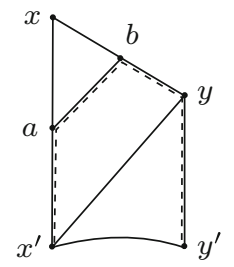

Case 2

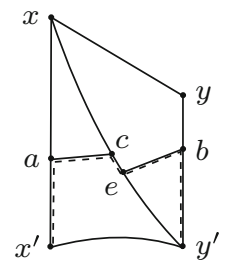

Case 3

Case $1 d\left(x, x^{\prime}\right) \leq 6 P$ : Define $\gamma:=\left[x^{\prime}, x\right]+[x, y]+\left[y, y^{\prime}\right]$. Then len $(\gamma) \leq 18 P<21 P$, so recurrence says there is a point $w \in \gamma$ such that $d\left(w, \mathcal{Z}^{\prime}\right) \leq D$. By $\left(^{*}\right)$, this is impossible.

Case $2 d\left(x, x^{\prime}\right)>6 P$ and $d\left(y, y^{\prime}\right) \leq 4 P$ : Let $a:=\left[x^{\prime}, x\right]\left(\frac{6 P}{d\left(x, x^{\prime}\right)}\right)$ and $b:=$ $[y, x]\left(\frac{6 P}{d\left(x, x^{\prime}\right)}\right)$, so that:

$$
d\left(a, x^{\prime}\right)=\frac{6 P}{d\left(x, x^{\prime}\right)} \cdot d\left(x, x^{\prime}\right)=6 P \quad \text { and } \quad d(b, y)=\frac{6 P}{d\left(x, x^{\prime}\right)} \cdot d(x, y) \leq 6 P
$$

Since $d\left(x^{\prime}, y\right) \leq 5 P$, the CAT $(0)$ condition implies $d(a, b)<5 P$. Define $\gamma:=\left[x^{\prime}, a\right]+$ $[a, b]+[b, y]+\left[y, y^{\prime}\right]$. Since len $(\gamma)<6 P+5 P+6 P+4 P=21 P$, recurrence says there is a point $w \in \gamma$ with $d\left(w, \mathcal{Z}^{\prime}\right) \leq D$. By $\left(^{*}\right)$, the only possibility is $w \in[a, b]$, but this is impossible because $d([a, b], \mathcal{Z}) \geq d(a, \mathcal{Z})-d(a, b)>6 P-5 P=P>D$.

Case $3 d\left(x, x^{\prime}\right)>6 P$ and $d\left(y, y^{\prime}\right)>4 P$ : Let $a:=\left[x^{\prime}, x\right]\left(\frac{4 P}{d\left(x, x^{\prime}\right)}\right)$ and let $c:=$ $\left[y^{\prime}, x\right]\left(\frac{4 P}{d\left(x, x^{\prime}\right)}\right)$. Then $d\left(x^{\prime}, a\right)=4 P$ and:

$$
4 P \leq d\left(y^{\prime}, c\right)=\frac{4 P}{d\left(x, x^{\prime}\right)} \cdot d\left(y^{\prime}, x\right) \leq \frac{4 P}{d\left(x, x^{\prime}\right)} \cdot\left(d\left(x, x^{\prime}\right)+P\right)<\frac{14}{3} P
$$

Let $b$ be the point of $\left[y^{\prime}, y\right]$ at distance $4 P$ from $y^{\prime}$, and let $e$ be the point of $\left[y^{\prime}, x\right]$ at distance $4 P$ from $y^{\prime}$, so $d(c, e)<\frac{2}{3} P$. The CAT(0) condition implies that $d(a, c)<P$ and, since $d(x, y) \leq d\left(x, y^{\prime}\right)$, that $d(e, b) \leq 4 \sqrt{2} P$.

Define $\gamma:=\left[x^{\prime}, a\right]+[a, c]+[c, e]+[e, b]+\left[b, y^{\prime}\right]$. Then len $(\gamma)<4 P+P+\frac{2}{3} P+$ $4 \sqrt{2} P+4 P<21 P$, so recurrence demands a point $w \in \gamma$ with $d\left(w, \mathcal{Z}^{\prime}\right) \leq D$. By $\left({ }^{*}\right)$, 
$w \notin\left[x^{\prime}, a\right],\left[b, y^{\prime}\right]$. We cannot have $w \in[a, c]+[c, e]$ because $d([a, c]+[c, e], \mathcal{Z}) \geq$ $d(a, \mathcal{Z})-(d(a, c)+d(c, e))>4 P-P-\frac{2}{3} P>D$. Thus, $w \in[e, b]$, so $d(e, b)=$ $d(e, w)+d(w, b)$. However, $d(w, b) \geq d(b, \mathcal{Z})-d(w, \mathcal{Z}) \geq 4 P-D>\frac{47}{12} P$. By the same reasoning, $\frac{47}{12} P<d(a, w)$, but $d(a, w)<P+\frac{2}{3} P+d(e, w)$, so $d(e, w)>\frac{27}{12} P$. This gives us the desired contradiction:

$$
6 P<\frac{74}{12} P<d(e, w)+d(w, b)=d(e, b) \leq 4 \sqrt{2} P<6 P
$$

Since all three cases ended in contradiction, we conclude $12 D$ bounds $\sigma$.

Acknowledgements Open access funding provided by Austrian Science Fund (FWF).

Open Access This article is distributed under the terms of the Creative Commons Attribution 4.0 International License (http://creativecommons.org/licenses/by/4.0/), which permits unrestricted use, distribution, and reproduction in any medium, provided you give appropriate credit to the original author(s) and the source, provide a link to the Creative Commons license, and indicate if changes were made.

\section{References}

1. Arzhantseva, G.N., Cashen, C.H., Gruber, D., Hume, D.: Characterizations of Morse geodesics via superlinear divergence and sublinear contraction. Doc. Math. 22, 1193-1224 (2017)

2. Arzhantseva, G.N., Cashen, C.H., Gruber, D., Hume, D.: Negative curvature in graphical small cancellation groups. Groups Geom. Dyn. 13(2), 579-632 (2019). https://doi.org/10.4171/GGD/498

3. Bridson, M.R., Haefliger, A.: Metric Spaces of Non-positive Curvature. Grundlehren der mathematischen Wissenschaften, vol. 319. Springer, Berlin (1999)

4. Cashen, C.H., Mackay, J.M.: A metrizable topology on the contracting boundary of a group. Trans. Amer. Math. Soc. (2019). https://doi.org/10.1090/tran/7544

5. Druţu, C., Mozes, S., Sapir, M.: Divergence in lattices in semisimple Lie groups and graphs of groups. Trans. Am. Math. Soc. 362(5), 2451-2505 (2010)

6. Genevois, A.: Hyperbolicities in CAT(0) cube complexes. Preprint (2017). arXiv:1709.08843

7. Russell, J., Spriano, D., Tran, H.C.: Convexity in hierarchically hyperbolic spaces. Preprint (2018). arXiv: 1809.09303

8. Sultan, H.: Hyperbolic quasi-geodesics in CAT(0) spaces. Geom. Dedicata 169(1), 209-224 (2014)

Publisher's Note Springer Nature remains neutral with regard to jurisdictional claims in published maps and institutional affiliations. 